\title{
Empirical Models of the Azimuthal Reception Angle- Part I: Comparative Analysis of Empirical Models for Different Propagation Environments
}

\author{
Cezary Ziółkowski ${ }^{1}$ (D) Jan M. Kelner $^{1}$ (D)
}

Published online: 21 July 2016

(C) The Author(s) 2016. This article is published with open access at Springerlink.com

\begin{abstract}
Statistical properties of the reception angle have a significant impact on the choice of the antenna system patterns and decide on the received signal-processing methods. For angle of arrival in azimuth plane, comparative analysis of the empirical models and the approximation error evaluation are the purpose of this paper. Here, the presented analysis is focused on models such as the von Mises, modified Gaussian, modified Laplacian, and modified logistic. For each model, the approximation accuracy is determined with respect to measurement data for seven different propagation scenarios. The measures such as the least-squares error, difference of standard deviations, Kolmogorov-Smirnov statistic, and Cramer-von Mises statistic are used for evaluation of the approximation errors. Comparative analysis for four empirical models, differentiation of propagation environments, multi-criterial evaluation of approximation errors in significant degree fill a gap in the previous analysis presented in the literature. The obtained results show that the empirical models provide a better fit to the measurement data than the geometrical models, and the smallest errors of approximation are for the modified Laplacian and logistic distributions.
\end{abstract}

Keywords Wireless communications - Multipath channel - Scattering - Angle of arrival (AOA) - probability density function (PDF) - Empirical models of PDF of azimuthal AOA . Laplacian · Gaussian · Logistic · von Mises distribution

Jan M. Kelner

jan.kelner@wat.edu.pl

Cezary Ziółkowski

cezary.ziolkowski@wat.edu.pl

1 Institute of Telecommunications, Faculty of Electronics, Military University of Technology,

Gen. Sylwester Kaliski Str. No. 2, 00-908 Warsaw, Poland 


\section{Introduction}

In multipath propagation environments, the reception angle spread is one of the main causes of received signal distortions. Statistical properties of the angle of arrival (AOA) are critical to select both the antenna system pattern and signal-processing method. Therefore, the probability density function (PDF) models of AOA are important for the design of antenna systems and theoretical and simulation studies of signals in wireless systems.

In practice, the theoretical (geometrical) and empirical models of PDF are used to reproduce the statistical properties of AOA. The first group of models is defined by geometrical structures that describe the spatial location of the scattering areas in 2D or 3D. The most commonly used geometrical structures are: circle [1-4], ellipse [3, 5-7], ring [8], hemisphere [9], cutting hemisphere [10], and cylinder [11-13]. Distribution of scatterers in propagation environment is an additional characteristic that defines each geometrical model. For these models, the following distributions are used: uniform [2, 4, 5, 8, 14], Gaussian [15, 16], Raleigh and exponential [2], hyperbolic [1], conical [17], parabolic $[3,18]$, and inverted parabolic [19]. The choice of the geometrical structure (shape, position, size) and scattering distribution determines the accuracy of the mapping of the actual propagation conditions.

The empirical models are based on the standard PDFs that are used in the calculus of probability. The adaptation of empirical model to different environment conditions involves selecting PDF parameter that minimizes the approximation error for measurement data. The advantage of these models is simplicity of the analytical form in which the spread of AOA is described by a single parameter.

In [20], the comparative analysis of the $2 \mathrm{D}$ geometrical models with respect to measurement data for different propagation scenarios is presented. However, this analysis applies only to the geometrical models. Therefore, in order to assess the effectiveness of PDF of AOA mapping by the various models, extending the comparative analysis on the empirical models it is reasoned. The results allow for a comparison of PDF approximation errors for the simple empirical and analytically complex geometrical models. The simplicity of the analytical description of PDF significantly simplifies the correlation and spectral analysis of the received signal. Therefore, the purpose of the comparative analysis is to assess and identify those empirical models that minimize PDF of AOA approximation error for the different propagation scenarios.

This paper focuses on statistical properties of the azimuthal AOA (AAOA). The presented analysis refers to wireless access systems, for which the propagation has a dominant role in azimuth plane. In practice, this means that the power pattern width is no more than a dozen degrees in elevation plane. In the first part of this paper, the main purpose is a comparative analysis and approximation error evaluation of the empirical models for different propagation environments. In [20], the obtained results show that none of the geometrical models provides minimization of the approximation error for all analyzed measurement scenarios. With regard to the empirical models, certain attempts to compare them are shown in [21]. However, the presented results do not provide a generalization of the conclusions because the single propagation scenario and only two models (truncated Laplacian and truncated Gaussian) are analyzed. In this paper, a wider analysis is presented. Here, the following models are considered: modified Gaussian, modified Laplacian, modified logistic, and von Mises. For each model, the approximation accuracy is evaluated with respect to measurement data from seven different propagation scenarios. In the literature, there are also other models such as the truncated cosine and 
uniform distribution in a limited angle range [22]. These models are not considered because their graphical representations are significantly different from the measurement data.

In this part of the paper, the comparative analysis of statistical AAOA models is based on the approximation error evaluation relative to measurement data. This error is determined by the classic measure that is the least-square error (LSE), and the statistical measures such as the standard deviation, Kolmogorov-Smirnov and Cramer-von Mises statistic. Thus, the obtained results provide a reliable assessment of the suitability of the different empirical models to represent the statistical properties of the signal reception angle in an azimuth plane. Comparative analysis of four models, a variety of propagation environments, and multi-criteria evaluation of approximation error largely fill the gaps in the existing analyses that are presented in the open literature.

In this paper, the presented results have a practical significance. They are the basis to select model and to evaluate its approximation error for different types of environments. Angular dispersion of the received signals depends on the type of propagation environment. Therefore, analytical and simulation studies need to clearly identify the model parameter. For each type of environment, the selection method of the model parameter is presented in Part II of this paper [23].

Part I of the paper is organized as follows. For description of the angle statistical properties, the method for modification of the standard PDF is presented in Sect. 2. Descriptions of the scenarios and the results that are reference data for empirical models are contained in Sect. 3. For particular models, the criteria and the evaluation of approximation errors are presented in Sect. 4. As a result of a comparative analysis, the empirical models, which accurately map the measurement data for the analyzed scenarios, are indicated in Sect. 5.

\section{Empirical Models of AAOA}

In the open literature, the statistical AAOA analysis shows that the empirical data approximation is based on PDF such as the Laplacian [15, 21, 22, 24-29], Gaussian [22, 24, 30, 31], and von Mises [22, 32-36]. In this paper, the logistic distribution [37-40]

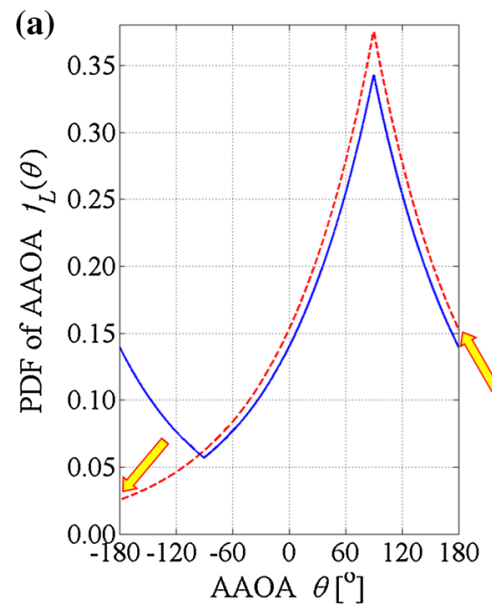

(b)

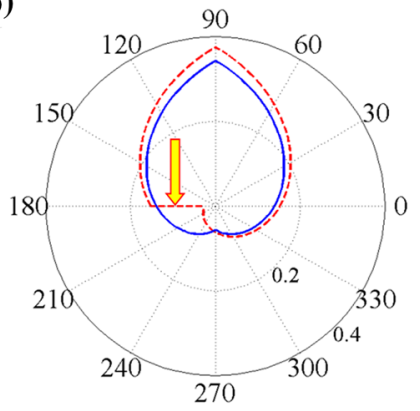

---Truncated Laplacian: $\theta_{0}=90^{\circ}, \lambda=0.01{ }^{\circ}$
-Modified Laplacian: $\theta_{0}=90^{\circ}, \lambda=0.01 \%^{\circ}$

Fig. 1 Differences between the truncated and modified Laplacian distributions in a Cartesian and $\mathbf{b}$ polar coordinate systems 
Table 1 Empirical models of PDF of AAOA $\left(\theta_{0} \in(-\pi, \pi\rangle\right)$

\section{Empirical model}

\section{Modified Gaussian}

Model parameter: $\sigma$

Normalized factor:

$C_{G}($ see (7))

\section{Modified Laplacian}

Model parameter: $\lambda$

Normalized factor:

$C_{L}($ see $(8))$

\section{Modified logistic}

Model parameter: $s$

Normalized factor:

$C_{S}$ (see (9))

von Mises

Model parameter: $\kappa$

$$
f_{M}(\theta)=\frac{\exp \left(\kappa \cos \left(\theta-\theta_{0}\right)\right)}{2 \pi \mathrm{I}_{0}(\kappa)} \quad \text { for } \quad \theta \in(-\pi, \pi\rangle
$$

For $\theta_{0}<0$

For $\theta_{0} \geq 0$

For $\theta_{0}<0$

For $\theta_{0} \geq 0$

$$
f_{S}(\theta)=\left\{\begin{array}{cl}
C_{S} \frac{\exp \left(-\frac{\theta-\theta_{0}+2 \pi}{s}\right)}{s\left(1+\exp \left(-\frac{\theta}{s}\right)\right)^{2}} & \text { for } \theta \in\left(-\pi, \theta_{0}-\pi\right\rangle \\
C_{S} \frac{\exp \left(-\frac{\theta-\theta_{0}}{s}\right)}{s\left(1+\exp \left(-\frac{\theta}{s}\right)\right)^{2}} & \text { for } \theta \in\left(\theta_{0}-\pi, \pi\right\rangle
\end{array}\right.
$$

For $\theta_{0}<0$

$$
f_{S}(\theta)=\left\{\begin{array}{c}
C_{S} \frac{\exp \left(-\frac{\theta-\theta_{0}}{s}\right)}{s\left(1+\exp \left(-\frac{\theta}{s}\right)\right)^{2}} \quad \text { for } \theta \in\left(-\pi, \theta_{0}+\pi\right\rangle \\
C_{S} \frac{\exp \left(-\frac{\theta-\theta_{0}-2 \pi}{s}\right)}{s\left(1+\exp \left(-\frac{\theta}{s}\right)\right)^{2}} \text { for } \theta \in\left(\theta_{0}+\pi, \pi\right\rangle
\end{array}\right.
$$

$$
f_{G}(\theta)= \begin{cases}C_{G} \frac{1}{\sqrt{2 \pi} \sigma} \exp \left(-\frac{\left(\theta-\theta_{0}+2 \pi\right)^{2}}{2 \sigma^{2}}\right) & \text { for } \theta \in\left(-\pi, \theta_{0}-\pi\right\rangle \\ C_{G} \frac{1}{\sqrt{2 \pi} \sigma} \exp \left(-\frac{\left(\theta-\theta_{0}\right)^{2}}{2 \sigma^{2}}\right) & \text { for } \theta \in\left(\theta_{0}-\pi, \pi\right\rangle\end{cases}
$$

$$
f_{G}(\theta)= \begin{cases}C_{G} \frac{1}{\sqrt{2 \pi} \sigma} \exp \left(-\frac{\left(\theta-\theta_{0}\right)^{2}}{2 \sigma^{2}}\right) & \text { for } \theta \in\left(-\pi, \theta_{0}+\pi\right\rangle \\ C_{G} \frac{1}{\sqrt{2 \pi} \sigma} \exp \left(-\frac{\left(\theta-\theta_{0}-2 \pi\right)^{2}}{2 \sigma^{2}}\right) & \text { for } \theta \in\left(\theta_{0}+\pi, \pi\right\rangle\end{cases}
$$

$$
f_{L}(\theta)= \begin{cases}C_{L} \frac{\lambda}{2} \exp \left(-\lambda\left|\theta-\theta_{0}+2 \pi\right|\right) & \text { for } \quad \theta \in\left(-\pi, \theta_{0}-\pi\right\rangle \\ C_{L} \frac{\lambda}{2} \exp \left(-\lambda\left|\theta-\theta_{0}\right|\right) & \text { for } \theta \in\left(\theta_{0}-\pi, \pi\right\rangle\end{cases}
$$

$$
f_{L}(\theta)= \begin{cases}C_{L} \frac{\lambda}{2} \exp \left(-\lambda\left|\theta-\theta_{0}\right|\right) & \text { for } \theta \in\left(-\pi, \theta_{0}+\pi\right\rangle \\ C_{L} \frac{\lambda}{2} \exp \left(-\lambda\left|\theta-\theta_{0}-2 \pi\right|\right) & \text { for } \theta \in\left(\theta_{0}+\pi, \pi\right\rangle\end{cases}
$$

$\mathrm{I}_{0}(\cdot)$ is the zero-order modified Bessel function 
is also taken into consideration because its graph is very similar to the measurement results. The range of AAOA limits the support of PDF to $(-\pi, \pi\rangle$ interval. For creating PDF with limited support, the commonly used method consists of the truncated standard PDF and the introduction of normalizing constant. However, for asymmetrical position of PDF extreme relative to the interval center $\left(\theta_{0} \neq 0\right.$, where $\theta_{0}$ is the average AAOA), the truncated PDF does not provide a mapping of AAOA real properties. In the polar coordinate system, this function is not continuity. Therefore, in the analytical description of the truncated distribution, the introduction of the additional modification is necessary. For example, the differences between the truncated and modified Laplacian distributions are presented in the Cartesian (Fig. 1a) and polar (Fig. 1b) coordinate systems.

The analytical relationships that define the empirical models of PDF such as the modified Gaussian, $f_{G}(\theta)$, modified Laplacian, $f_{L}(\theta)$, modified logistic, $f_{S}(\theta)$, and von Mises, $f_{M}(\theta)$ are shown in Table 1 .

Minimization of the approximation error is a criterion to fit the model to measurement data. The ranges of the model parameters are determined by the boundary conditions of reception angle. These conditions are defined by the standard deviation, $\sigma_{\theta}$, which is a measure of real angle dispersion. For the case of maximum reception angle concentration (receiving direct path), PDF approaches delta distribution. In practice, a finite accuracy of measurements is the basis to adopt $\sigma_{\theta}=1^{\circ}$. For the von Mises distribution, this value is obtained for $\kappa \rightarrow \infty$. Because of numerical calculation complexity of the Bessel function, the following approximation is used [41]:

$$
\lim _{\kappa \rightarrow \infty} \mathrm{I}_{0}(\kappa) \cong \frac{\exp (\kappa)}{\sqrt{2 \pi \kappa}}
$$

For this case, the von Mises PDF is

$$
\lim _{\kappa \rightarrow \infty} f_{M}(\theta) \cong \sqrt{\frac{\kappa}{2 \pi}} \exp (\kappa(\cos (\theta)-1))
$$

For maximum spread of the reception angle, PDF of AAOA tends to uniform distribution, that is $\sigma_{\theta}=103^{\circ}$. For the reception boundary conditions, the ranges of parameters, $\sigma, \lambda, s, \kappa$ for the modified Gaussian, modified Laplacian, modified logistic, and von Mises distributions are contained in Table 2.

The modified Gaussian, Laplacian, and logistic PDF require the introduction of the normalized factors, $C_{G}(\sigma), C_{L}(\lambda), C_{S}(s)$, respectively

Table 2 The ranges of the parameters for empirical models

\begin{tabular}{llll}
\hline Empirical model & Model parameter & \multicolumn{2}{l}{ Boundary values of model parameter } \\
\cline { 3 - 4 } & & $\sigma_{\theta}=1^{\circ}$ & $\sigma_{\theta}=103^{\circ}$ \\
\hline Modified Gaussian & $\sigma\left(^{\circ}\right)$ & 1.0 & 492 \\
Modified Laplacian & $\lambda\left(1 /^{\circ}\right)$ & 1.4 & 0.00039 \\
Modified logistic & $s\left(^{\circ}\right)$ & 0.56 & 347 \\
von Mises & $\kappa(1)$ & 3283 & $\approx 0$ \\
\hline
\end{tabular}




$$
\begin{gathered}
C_{G}=\frac{1}{\int_{-\pi}^{\pi} f_{G}(\theta) \mathrm{d} \theta}=\frac{1}{\operatorname{erf}\left(\frac{\pi}{\sqrt{2} \sigma}\right)} \\
C_{L}=\frac{1}{\int_{-\pi}^{\pi} f_{L}(\theta) \mathrm{d} \theta}=\frac{1}{1-\exp (-\lambda \pi)} \\
C_{S}=\frac{1}{\int_{-\pi}^{\pi} f_{S}(\theta) \mathrm{d} \theta}=\frac{\left(1+\exp \left(\frac{\pi}{s}\right)\right)\left(1+\exp \left(-\frac{\pi}{s}\right)\right)}{\exp \left(\frac{\pi}{s}\right)-\exp \left(-\frac{\pi}{s}\right)}
\end{gathered}
$$

where $\operatorname{erf}(\cdot)$ is the error function. These factors depend on parameters of particular PDFs. The normalized factors versus parameters of analyzed PDFs are contained in Table 1, whereas their graphical presentations are shown in Fig. 2.

The graphs show that the normalized factors are approximately equal to 1 for specified range of parameters of analyzed PDFs. For particular models, $C_{G} \cong 1.000$ for $\sigma \leq 44^{\circ}$, $C_{L} \cong 1.000$ for $\lambda \geq 0.056 /{ }^{\circ}$, and $C_{S} \cong 1.000$ for $s \leq 23^{\circ}$.

The angle spread of the received signals depends on the type of propagation environment. The empirical model adaptation to different environment conditions consists of such matching of its parameter that provides the best fit to the real PDF of AOA. For analyzed models, the graphical representations of parameter influence on the mapping of the reception angle spread are presented in Fig. 3.

The graphs show that the appropriate choice of PDF parameter enables the model fit to the measurement data.

(a)

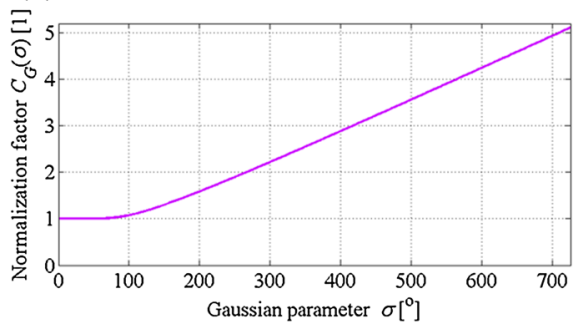

(b)

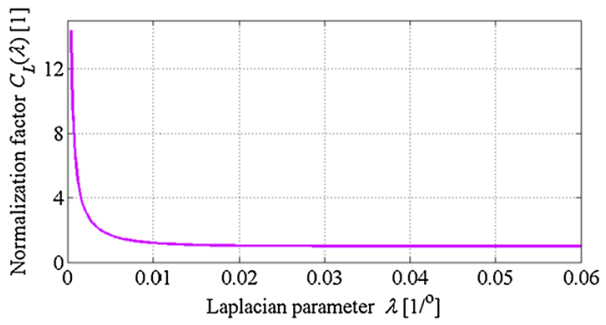

(c)

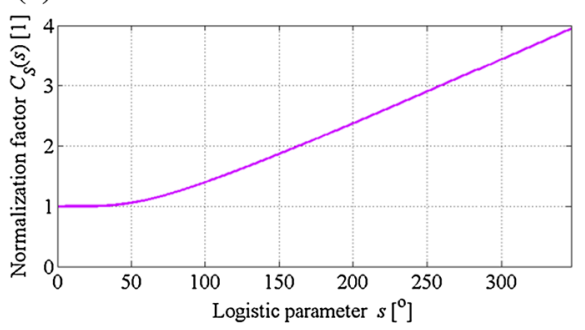

Fig. 2 Normalized factor versus model parameter for the modified distributions: a Gaussian, b Laplacian, c logistic 
(a)

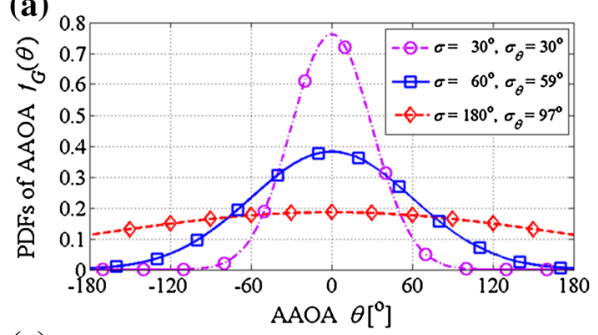

(c)

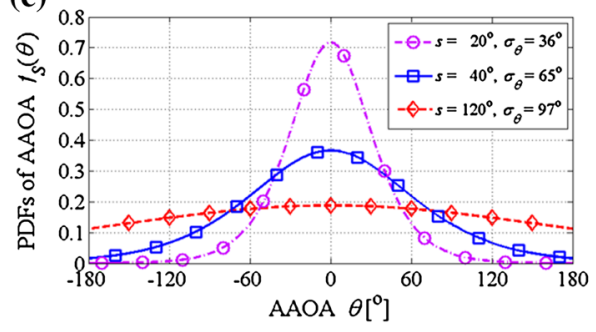

(b)

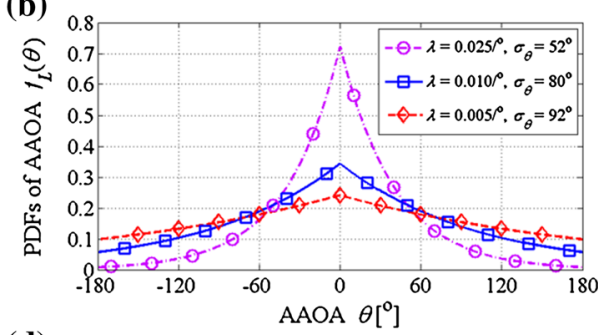

(d)

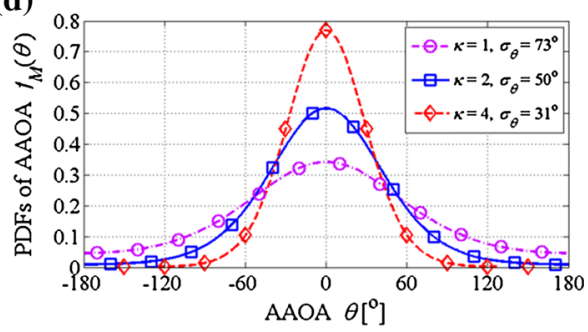

Fig. 3 Influence of the model parameter on the mapping of reception angle spread for the following PDFs: a modified Gaussian, b modified Laplacian, c) modified logistic, $\mathbf{d}$ von Mises

\section{Measurement Scenarios}

Presented in [20], a review and evaluation of the theoretical models of PDF of AAOA are the basis for the selection of the measurement scenarios for comparative analysis of empirical models. In this part of the paper, the comparative analysis is focused on scenarios that concern the homogeneous propagation environments. With respect to empirical PDF, it means a unimodal function type. Compared to [20], here the set of measurement results is limited to seven scenarios that are described in [21, 42-46]. Out of all unimodal, empirical PDFs that are included in [20], the measurement data from [47] are not contained in this paper. For this case, the correction changing position of transmitter (Tx) is not included in AAOA averaging procedure.

The choice of the measurement scenarios provides the differentiation of the analyzed propagation environments. Reference data are derived from the measurements that have been made in both rural environment [43] as well as urban environment with different density of buildings [21, 42, 44-46]. The type of propagation environment is determined by the height of the receiving antenna location relative to the mean height of buildings. For [42, 45, 46] and [21] (Aarhus) scenarios, the location height of antennas significantly exceed the mean height of buildings. As a result, the propagation conditions correspond to the typical urban environment type. Some important factors that affect the wave propagation are the distance between $\mathrm{Tx}$ and receiver $(\mathrm{Rx})$, and a carrier frequency of the sounding signal. For particular scenarios, these data are included in Table 3.

For the macro- and microcell, the measure that defines the type of propagation environment is the rms delay spread (DS) 


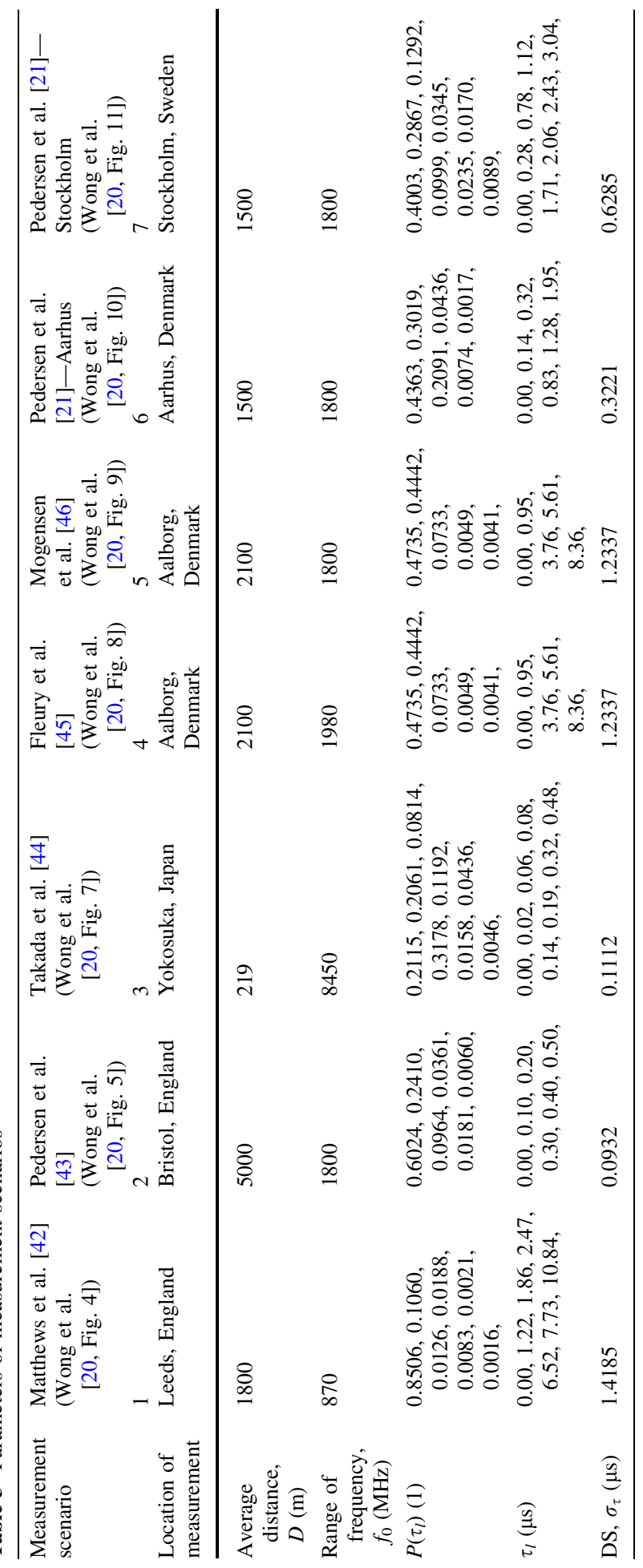


Table 4 Optimal parameters of empirical models for individual scenarios

\begin{tabular}{lllllllll}
\hline Empirical model & $\begin{array}{l}\text { Model } \\
\text { parameter }\end{array}$ & \multicolumn{2}{l}{ Measurement scenario } & & & & \\
\cline { 3 - 9 } & & 1 & 2 & 3 & 4 & 5 & 6 & 7 \\
\hline $\begin{array}{c}\text { Modified } \\
\text { Gaussian }\end{array}$ & $\sigma\left(^{\circ}\right)$ & 7.952 & 1.170 & 1.918 & 6.018 & 5.570 & 4.133 & 7.673 \\
$\begin{array}{c}\text { Modified } \\
\text { Laplacian }\end{array}$ & $\lambda\left(1 /^{\circ}\right)$ & 0.125 & 0.844 & 0.620 & 0.167 & 0.174 & 0.249 & 0.135 \\
$\begin{array}{c}\text { Modified } \\
\text { Logistic }\end{array}$ & $s\left(^{\circ}\right)$ & 4.922 & 0.720 & 1.129 & 3.732 & 3.460 & 2.494 & 4.649 \\
von Mises & $\kappa(1)$ & 52.2 & 2398.4 & 893.5 & 90.9 & 106.1 & 192.5 & 56.0 \\
\hline
\end{tabular}

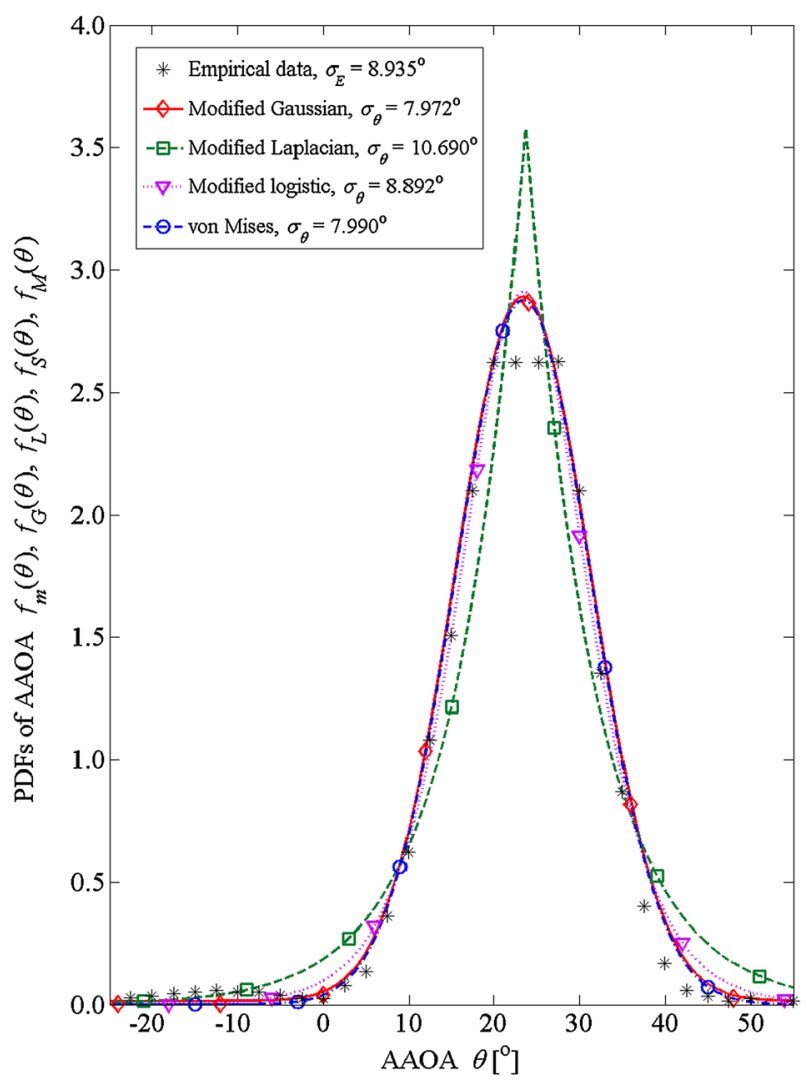

Fig. 4 Scenario 1. Matthews et al. [42]—Leeds

$$
\sigma_{\tau}=\sqrt{\frac{\int_{0}^{\infty} \tau^{2} P(\tau) \mathrm{d} \tau}{\int_{0}^{\infty} P(\tau) \mathrm{d} \tau}-\left(\frac{\int_{0}^{\infty} \tau P(\tau) \mathrm{d} \tau}{\int_{0}^{\infty} P(\tau) \mathrm{d} \tau}\right)^{2}}
$$


where $P(\tau)$ is the power delay profile (PDP) or power delay spectrum (PDS).

PDP and PDS are the basis for numerical calculation of DS. In Table 3, these functions are presented in the form of a discrete set of $\tau_{l}$ and $P\left(\tau_{l}\right)$.

The diversity of DSs shows the different types of propagation environments such as rural, suburban, and urban areas with sparse and dense buildings. The antenna heights of Tx and Rx relative to the average height of the buildings is the main factor, which decides about diversity propagation conditions. In [43] (scenario 2), time characteristics such as PDS or PDP are not included in measurement data set. In this case, the descriptions of the propagation environment and measurement conditions are utilized. Hence, to assess the type of propagation environment, $\tau_{l}$ and $P\left(\tau_{l}\right)$ are adopted according to COST 207 for rural area [48].

\section{Comparative Analysis of Empirical Models}

The assessment of the approximation error is the basis for comparative analysis of empirical models of PDF of AAOA for different environmental conditions. Here, as in [20], LSE is used as a measure of goodness of fit of the model to the measurement data

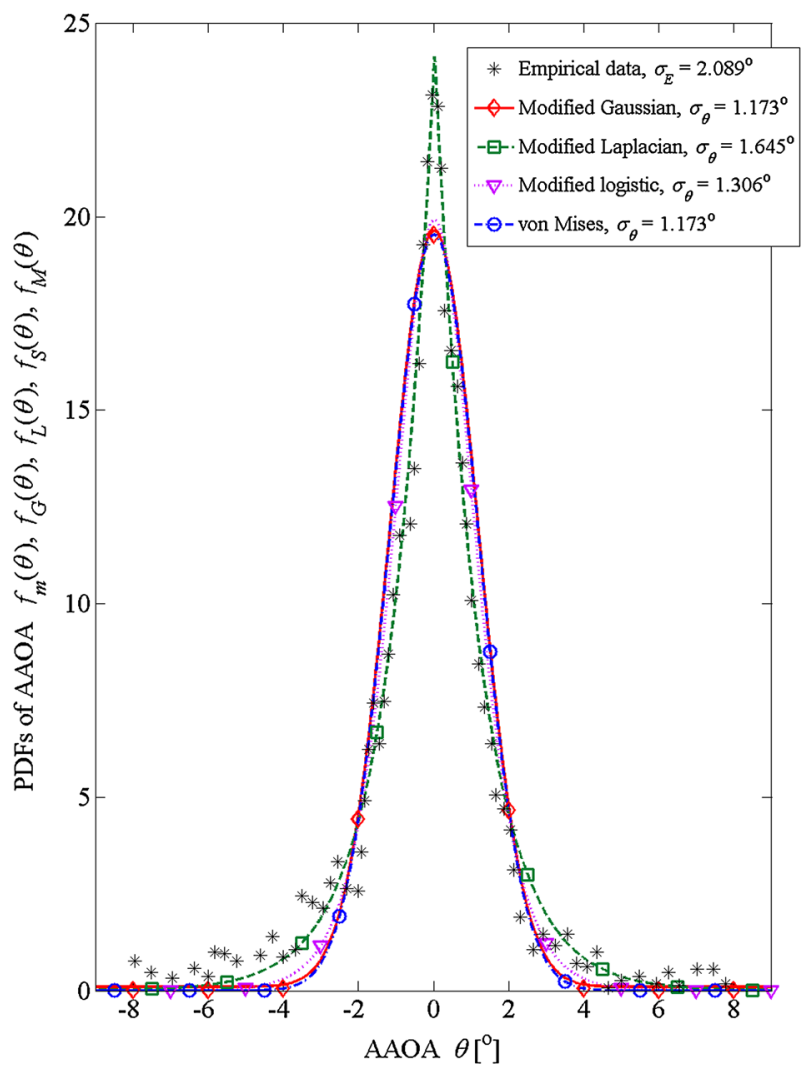

Fig. 5 Scenario 2. Pedersen et al. [43]-Bristol 


$$
L S E=\frac{1}{K} \sum_{k=1}^{K}\left[f_{E}\left(\theta_{k}\right)-f\left(\theta_{k}\right)\right]^{2}
$$

where $f_{E}\left(\theta_{k}\right)(k=1, \ldots, K)$ denotes the normalized values from empirical dataset, $K$ refers to the cardinality of the set of measurement data, and $f\left(\theta_{k}\right)$ represents the values for the analyzed empirical model of PDF.

The application of this measure gives the opportunity to use the results from [20] to compare the empirical models and the best geometrical models against the measured data. LSE minimize is also used as a criterion for matching model parameters to measurement data for the particular propagation scenarios. The empirical model parameters that minimize the approximation errors of measurement data are included in Table 4, whereas the graphical representations of PDFs are shown in Figs. 4, 5, 6, 7, 8, 9 and 10. For the empirical PDFs, the standard deviation is marked as $\sigma_{E}$.

The empirical data are obtained on the basis of measurement data graphs that are presented in [20]. To extract the numeric data, the software WebPlotDigitizer is applied [49].

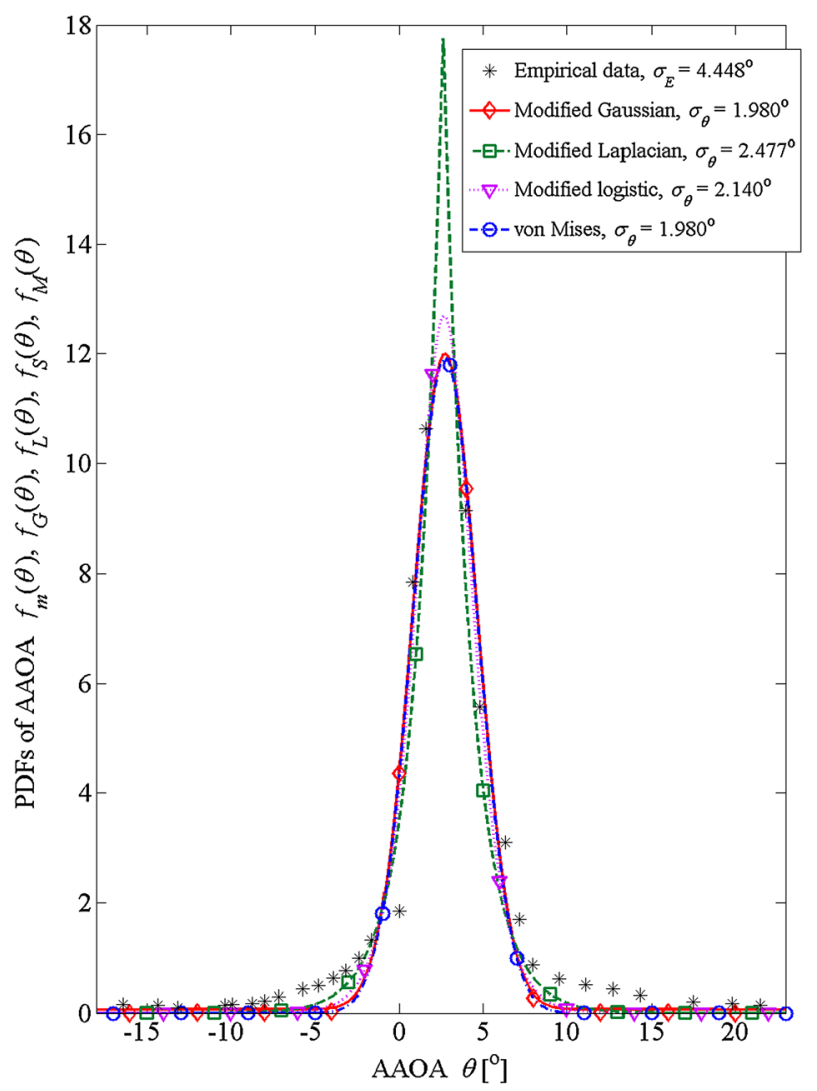

Fig. 6 Scenario 3. Takada et al. [44]-Yokosuka 


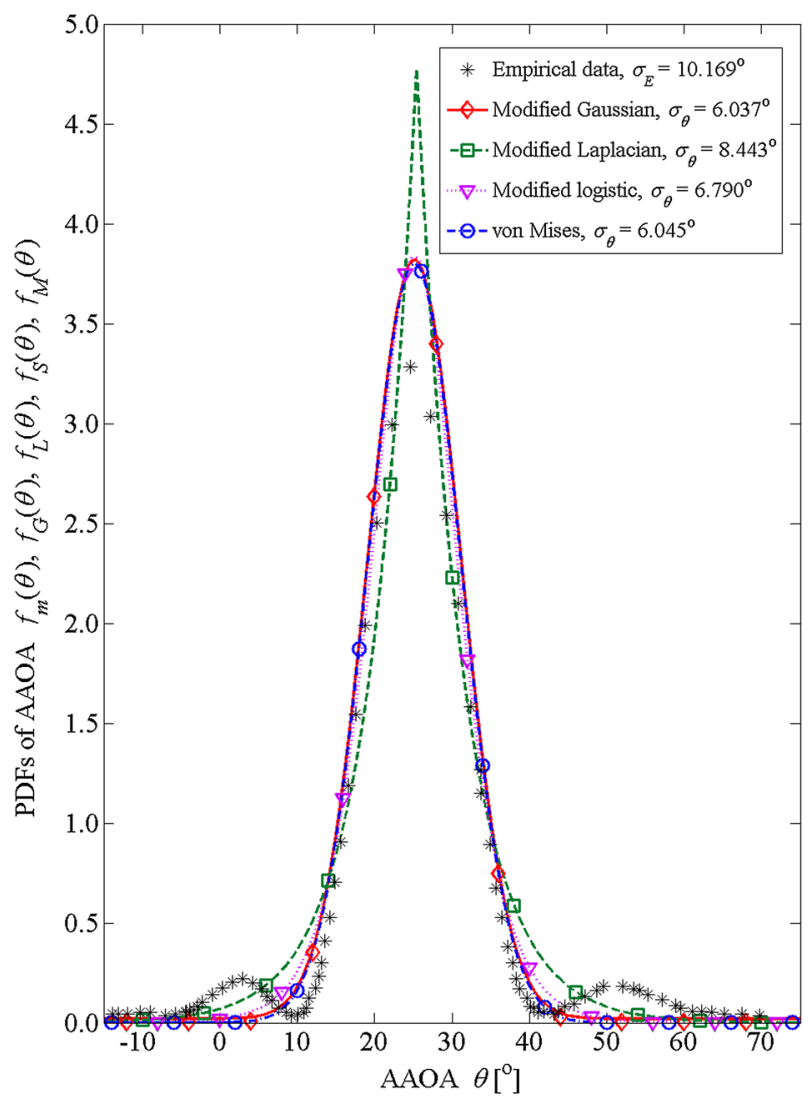

Fig. 7 Scenario 4. Fleury et al. [45]—Aalborg

For the analyzed scenarios, LSE is the basis for the fit accuracy evaluation of the empirical models to measurement data. LSE is calculated for optimal parameters, and the obtained results are included in Table 5. In [20], the same measure is used to assess the approximation accuracy of the geometrical models. This gives us the opportunity to compare the approximation errors for empirical and theoretical models of PDF of AAOA. Therefore, the smallest approximation errors for the geometrical models from [20] are also included in Table 5.

For different measuring scenarios, the obtained results show that the uniform elliptical model (Rx outside) provides the smallest LSE in a group of the geometrical models. But a more accurate mapping of measurement data can be obtained by making use of the modified Laplacian model, which belongs to the simple empirical models. In general, the results in the Table 5 show that in addition to the uniform elliptical model (Rx outside), all the geometrical models give greater approximation errors as compared to the empirical models.

In this paper, the presented comparative analysis concerns the statistical functions that described the properties of AAOA. Therefore, the statistical measures should be used to assess the accuracy of PDF approximation. 


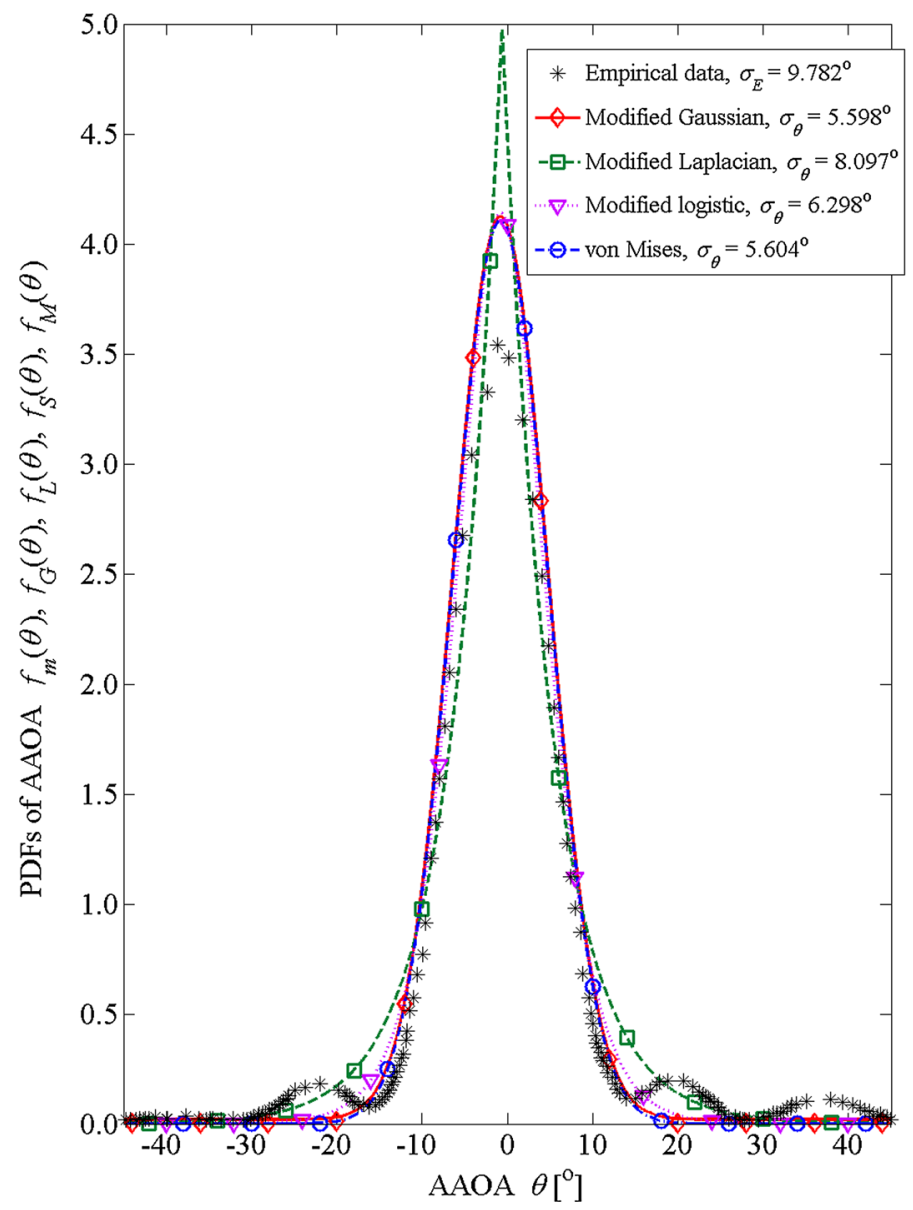

Fig. 8 Scenario 5. Mogensen et al. [46]—Aalborg

The basic measure, $\Delta \sigma$, is based on a comparison of the standard deviations for model and measurement data

$$
\Delta \sigma=\left|\sigma_{E}-\sigma_{\theta}\right|
$$

where $\sigma_{E}, \sigma_{\theta}$ are the standard deviations for measurement data and PDF model, respectively. These parameters are also called the rms azimuth angle spread, when they are determined on the basis of the power azimuth spectrum.

For the analyzed scenarios and PDF models, the comparative analysis results based on $\Delta \sigma$ are included in Table 6.

However, the application of $\Delta \sigma$ is limited only to assess the dispersion degree of PDFs. Therefore, the similarity assessment of PDFs requires the use of dedicated statistical measures such as the Cramer-von Mises and Kolmogorov-Smirnov statistics [55]. These statistics are described by, respectively 


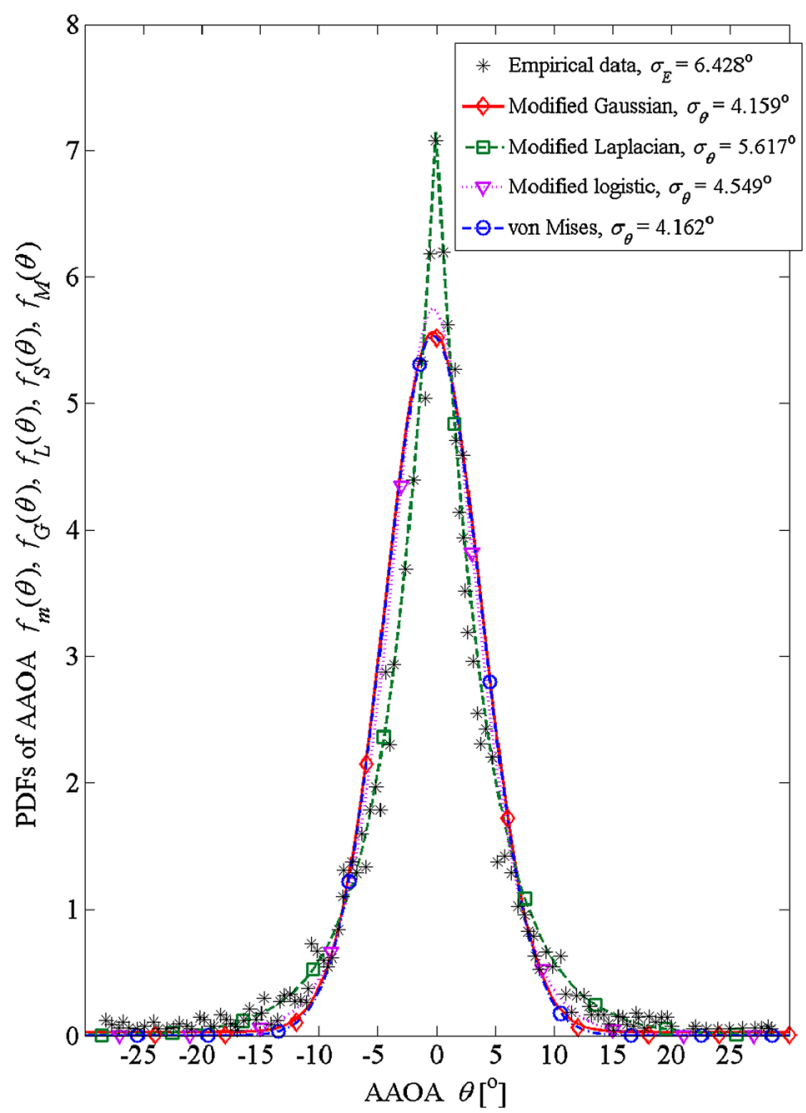

Fig. 9 Scenario 6. Pedersen et al. [21]—Aarhus

$$
\begin{gathered}
W^{2}=\int_{-\pi}^{\pi}\left[F_{E}(\theta)-F(\theta)\right]^{2} \mathrm{~d} F(\theta) \\
D_{n}=\sup _{\theta}\left|F_{E}(\theta)-F(\theta)\right|
\end{gathered}
$$

where $F_{E}(\theta)=\int_{-\pi}^{\theta} f_{E}(\varphi) \mathrm{d} \varphi$ is the cumulative distribution function (CDF) for the PDF based on the empirical data, and $F(\theta)=\int_{-\pi}^{\theta} f(\varphi) \mathrm{d} \varphi$ is CDF for the analyzed empirical PDF model.

Based on (13) and (14), the obtained results of the numerical calculations are included in Tables 7 and 8, respectively.

For each PDF model, to assess the accuracy of the approximation, the average values of the utilized measures are included in the last column of each table. Table 5 shows that 


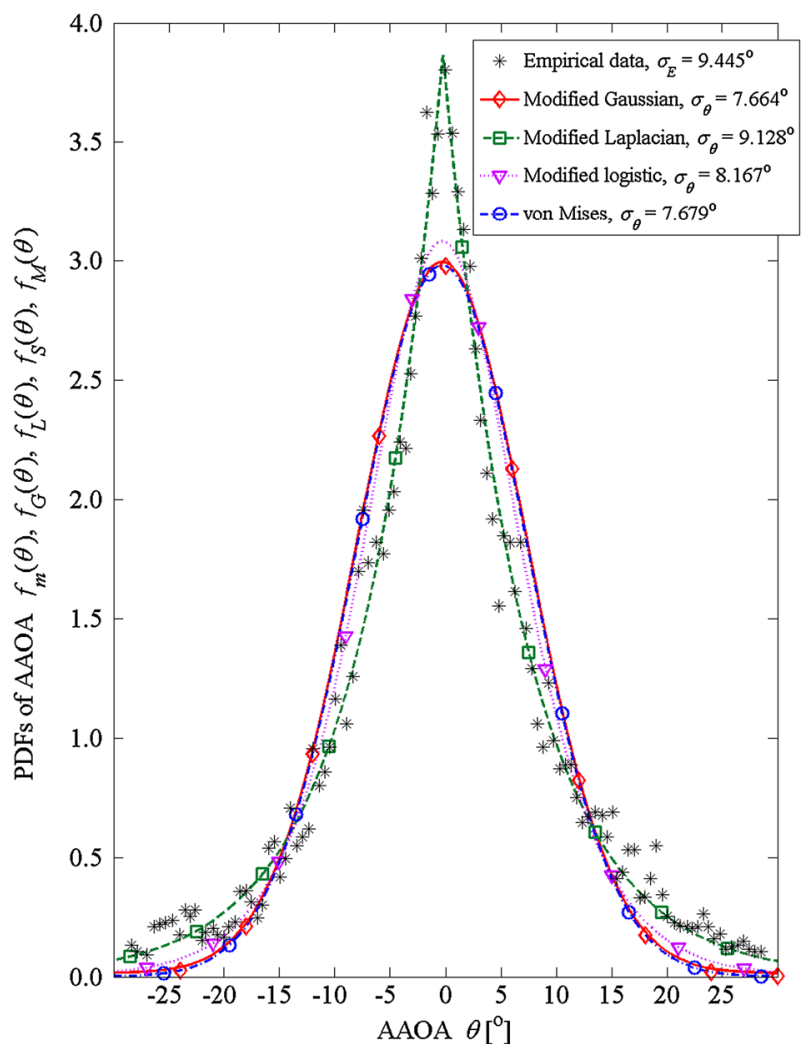

Fig. 10 Scenario 7. Pedersen et al. [21]-Stockholm

the empirical models generally provide a better fit to the measurement data than the geometrical models. Only the uniform elliptical ( $\mathrm{Rx}$ outside) and Gaussian models provide the results similar to the empirical models. Furthermore, for empirical models, the simplicity of analytical description is a significant prerequisite for the use of these models to assess AAOA statistical properties in analytical and simulation studies. Tables 6, 7 and 8 show that for all statistical measures, Laplacian and logistic models provide the smallest approximation error for large number of measuring scenarios. When choosing the best model, ambiguity occurs only for scenarios 1 and 3. Unlike other scenarios, the measurement results were obtained for the fixed positions of Tx and Rx. Thus, the empirical data from Leeds [42] and Yokosuka [44] do not account for the spatial averaging of measurements. This means that the evaluation of PDF of AAOA requires not only defining the model, type of environment, but also the choice of an appropriate measure for measurement campaign. The obtained results show that for a pair of the modified Laplacian-modified logistic and the modified Gaussian-von Mises models, convergence of the approximation errors occurs. Thus, these models can be used interchangeably for each pair. 


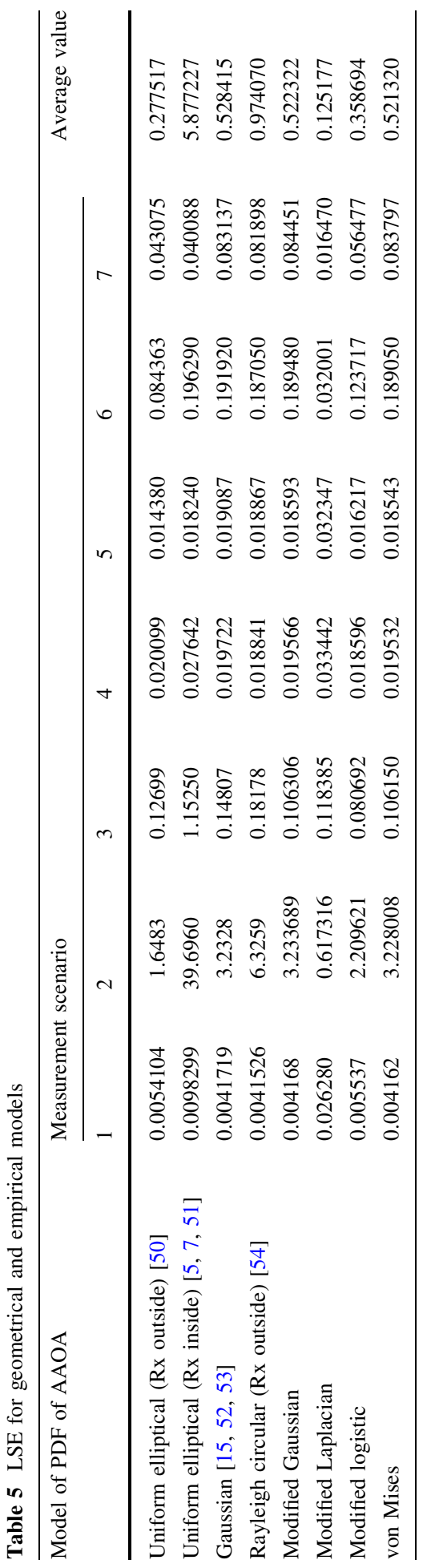


Table 6 Results of the comparative analysis based on $\Delta \sigma$

\begin{tabular}{lllllllll}
\hline Empirical model & \multicolumn{9}{l}{ Measurement scenario } & & & Average value \\
\cline { 2 - 7 } & 1 & 2 & 3 & 4 & 5 & 6 & 7 & \\
\hline Modified Gaussian & 0.963 & 0.916 & 2.468 & 4.132 & 4.184 & 2.269 & 1.781 & 2.388 \\
Modified Laplacian & 1.755 & 0.444 & 1.971 & 1.726 & 1.685 & 0.811 & 0.317 & 1.244 \\
Modified logistic & 0.043 & 0.783 & 2.308 & 3.379 & 3.484 & 1.879 & 1.278 & 1.879 \\
von Mises & 0.945 & 0.916 & 2.468 & 4.124 & 4.178 & 2.266 & 1.766 & 2.380 \\
\hline
\end{tabular}

Table 7 Results of the comparative analysis based on the Cramer-von Mises statistic

\begin{tabular}{|c|c|c|c|c|c|c|c|c|}
\hline \multirow{2}{*}{$\begin{array}{l}\text { Empirical } \\
\text { model }\end{array}$} & \multicolumn{7}{|c|}{ Measurement scenario } & \multirow{2}{*}{$\begin{array}{l}\text { Average } \\
\text { value }\end{array}$} \\
\hline & 1 & 2 & 3 & 4 & 5 & 6 & 7 & \\
\hline $\begin{array}{l}\text { Modified } \\
\text { Gaussian }\end{array}$ & 0.005888 & 0.106933 & 0.103394 & 0.040058 & 0.089772 & 0.070401 & 0.048080 & 0.066361 \\
\hline $\begin{array}{l}\text { Modified } \\
\text { Laplacian }\end{array}$ & 0.025826 & 0.052498 & 0.160256 & 0.028138 & 0.029616 & 0.018224 & 0.003668 & 0.045461 \\
\hline $\begin{array}{l}\text { Modified } \\
\text { logistic }\end{array}$ & 0.008097 & 0.085342 & 0.121122 & 0.023961 & 0.053179 & 0.043446 & 0.030093 & 0.052177 \\
\hline von Mises & 0.005918 & 0.106852 & 0.103283 & 0.039815 & 0.089315 & 0.070177 & 0.047527 & 0.066127 \\
\hline
\end{tabular}

Table 8 Results of the comparative analysis based on the Kolmogorov-Smirnov statistic

\begin{tabular}{|c|c|c|c|c|c|c|c|c|}
\hline \multirow{2}{*}{$\begin{array}{l}\text { Empirical } \\
\text { model }\end{array}$} & \multicolumn{7}{|c|}{ Measurement scenario } & \multirow{2}{*}{$\begin{array}{l}\text { Average } \\
\text { value }\end{array}$} \\
\hline & 1 & 2 & 3 & 4 & 5 & 6 & 7 & \\
\hline $\begin{array}{l}\text { Modified } \\
\text { Gaussian }\end{array}$ & 0.021816 & 0.089507 & 0.096766 & 0.049073 & 0.051052 & 0.047000 & 0.046356 & 0.057367 \\
\hline $\begin{array}{l}\text { Modified } \\
\text { Laplacian }\end{array}$ & 0.058585 & 0.044165 & 0.113555 & 0.032925 & 0.025971 & 0.020886 & 0.012385 & 0.044067 \\
\hline $\begin{array}{r}\text { Modified } \\
\text { logistic }\end{array}$ & 0.032103 & 0.075160 & 0.100261 & 0.043378 & 0.042719 & 0.035806 & 0.035452 & 0.052126 \\
\hline von Mises & 0.022023 & 0.089484 & 0.096701 & 0.049017 & 0.050963 & 0.046909 & 0.046037 & 0.057305 \\
\hline
\end{tabular}

\section{Conclusion}

In this paper, the comparative analysis of PDF empirical models has been performed based on approximation accuracy evaluation of the reception angle distribution to the measurement data for different propagation environments. The obtained results are an extension of comparative analysis that is presented in [20] and focus only on geometrical models.

Here, the classical PDFs are used to determine the empirical models of AAOA distributions. However, the mapping of the statistical properties of AAOA requires appropriate 
modification of these PDFs. The modifications, which are introduced into the classical models such as Gaussian, Laplacian, and logistic ensure the continuity of PDFs in polar coordinates. In physical interpretation, it provides the convergence of the measurement data and empirical models. For empirical and geometrical models, the comparison of approximation errors shows that simple empirical models fit better with the measurement data, whereas for most of the analyzed scenarios, the modified Laplacian and modified logistic models are best adopted to the empirical data. The obtained results show that minimizing the approximation error requires the selection and fitting of the model to the propagation environment type. The problem of the model adaptation to research scenario, that is, matching of model parameter to the type of propagation environment is essential for theoretical and simulation studies. This issue is examined in Part II of this paper [23].

Open Access This article is distributed under the terms of the Creative Commons Attribution 4.0 International License (http://creativecommons.org/licenses/by/4.0/), which permits unrestricted use, distribution, and reproduction in any medium, provided you give appropriate credit to the original author(s) and the source, provide a link to the Creative Commons license, and indicate if changes were made.

\section{References}

1. Le, K. N. (2009). On angle-of-arrival and time-of-arrival statistics of geometric scattering channels. IEEE Transactions on Vehicular Technology, 58(8), 4257-4264. doi:10.1109/TVT.2009.2023255.

2. Jiang, L., \& Tan, S. Y. (2007). Geometrically based statistical channel models for outdoor and indoor propagation environments. IEEE Transactions on Vehicular Technology, 56(6), 3587-3593. doi:10. 1109/TVT.2007.901055.

3. Chen, Y., \& Dubey, V. K. (2004). Accuracy of geometric channel-modeling methods. IEEE Transactions on Vehicular Technology, 53(1), 82-93. doi:10.1109/TVT.2003.821999.

4. Petrus, P., Reed, J. H., \& Rappaport, T. S. (2002). Geometrical-based statistical macrocell channel model for mobile environments. IEEE Transactions on Communications, 50(3), 495-502. doi:10.1109/ 26.990911.

5. Ertel, R. B., \& Reed, J. H. (1999). Angle and time of arrival statistics for circular and elliptical scattering models. IEEE Journal on Selected Areas in Communications, 17(11), 1829-1840. doi:10. $1109 / 49.806814$.

6. Liberti, J. C., \& Rappaport, T. S. (1999). Smart antennas for wireless communications: IS-95 and third generation CDMA applications. Upper Saddle River, NJ: Prentice Hall PTR.

7. Khan, N. M., Simsim, M. T., \& Rapajic, P. B. (2008). A generalized model for the spatial characteristics of the cellular mobile channel. IEEE Transactions on Vehicular Technology, 57(1), 22-37. doi:10.1109/ TVT.2007.904532.

8. Olenko, A. Y., Wong, K. T., \& Ng, E. H.-O. (2003). Analytically derived TOA-DOA statistics of uplink/downlink wireless multipaths arisen from scatterers on a hollow-disc around the mobile. IEEE Antennas and Wireless Propagation Letters, 2(1), 345-348. doi:10.1109/LAWP.2004.824174.

9. Janaswamy, R. (2002). Angle of arrival statistics for a 3-D spheroid model. IEEE Transactions on Vehicular Technology, 51(5), 1242-1247. doi:10.1109/TVT.2002.801756.

10. Nawaz, S. J., Khan, N. M., Patwary, M. N., \& Moniri, M. (2011). Effect of directional antenna on the Doppler spectrum in 3-D mobile radio propagation environment. IEEE Transactions on Vehicular Technology, 60(7), 2895-2903. doi:10.1109/TVT.2011.2161788.

11. Zajic, A. G., \& Stuber, G. L. (2008). Three-dimensional modeling, simulation, and capacity analysis of space-time correlated mobile-to-mobile channels. IEEE Transactions on Vehicular Technology, 57(4), 2042-2054. doi:10.1109/TVT.2007.912150.

12. Olenko, A. Y., Wong, K. T., \& Qasmi, S. A. (2013). Distribution of the uplink multipaths' arrival delay and azimuth-elevation arrival angle because of "bad urban" scatterers distributed cylindrically above the mobile. Transactions on Emerging Telecommunications Technologies, 24(2), 113-132. doi:10.1002/ ett.2530.

13. Zajic, A. G. (2014). Impact of moving scatterers on vehicle-to-vehicle narrow-band channel characteristics. IEEE Transactions on Vehicular Technology, 63(7), 3094-3106. doi:10.1109/TVT.2014. 2299239. 
14. Zekavat, S. A., \& Nassar, C. R. (2003). Power-azimuth-spectrum modeling for antenna array systems: a geometric-based approach. IEEE Transactions on Antennas and Propagation, 51(12), 3292-3294. doi:10.1109/TAP.2003.820973.

15. Janaswamy, R. (2002). Angle and time of arrival statistics for the Gaussian scatter density model. IEEE Transactions on Wireless Communications, 1(3), 488-497. doi:10.1109/TWC.2002.800547.

16. Wu, Y. I., \& Wong, K. T. (2011). Polarisation-sensitive geometric modelling of the distribution of direction-of-arrival for uplink multipaths. IET Microwaves, Antennas Propagation, 5(1), 95-101. doi:10.1049/iet-map.2010.0077.

17. Eggers, P. C. F. (1998). Generation of base station DOA distributions by Jacobi transformation of scattering areas. Electronics Letters, 34(1), 24-26. doi:10.1049/el:19980043.

18. Chen, Y., \& Dubey, V. K. (2005). Parabolic distribution of scatterers for street-dominated mobile environments. IEEE Transactions on Vehicular Technology, 54(1), 1-8. doi:10.1109/TVT.2004. 838846.

19. Olenko, A. Y., Wong, K. T., \& Abdulla, M. (2005). Analytically derived TOA-DOA distributions of uplink/downlink wireless-cellular multipaths arisen from scatterers with an inverted-parabolic spatial distribution around the mobile. IEEE Signal Processing Letters, 12(7), 516-519. doi:10.1109/LSP.2005. 847859.

20. Wong, K. T., Wu, Y. I., \& Abdulla, M. (2010). Landmobile radiowave multipaths' DOA-distribution: Assessing geometric models by the open literature's empirical datasets. IEEE Transactions on Antennas and Propagation, 58(3), 946-958. doi:10.1109/TAP.2009.2037698.

21. Pedersen, K. I., Mogensen, P. E., \& Fleury, B. H. (2000). A stochastic model of the temporal and azimuthal dispersion seen at the base station in outdoor propagation environments. IEEE Transactions on Vehicular Technology, 49(2), 437-447. doi:10.1109/25.832975.

22. Sieskul, B. T., Kupferschmidt, C., \& Kaiser, T. (2011). Spatial fading correlation for local scattering: A condition of angular distribution. IEEE Transactions on Vehicular Technology, 60(3), 1271-1278. doi:10.1109/TVT.2010.2103370.

23. Ziółkowski, C., \& Kelner, J. M. (in review). Empirical models of the azimuthal reception angle-Part II: Adaptation of the models to simulation studies-error analysis. Wireless Personal Communications.

24. Pedersen, K. I., Mogensen, P. E., \& Fleury, B. H. (1997). Power azimuth spectrum in outdoor environments. Electronics Letters, 33(18), 1583-1584. doi:10.1049/el:19971029.

25. Mucchi, L., Staderini, C., Ylitalo, J., \& Kyösti, P. (2007). Modified spatial channel model for MIMO wireless systems. EURASIP Journal on Wireless Communications and Networking, 2007(1), 068512. doi:10.1155/2007/68512.

26. Ichitsubo, S., Tsunekawa, K., \& Ebine, Y. (2002). Multipath propagation model of spatio-temporal dispersion observed at base station in urban areas. IEEE Journal on Selected Areas in Communications, 20(6), 1204-1210. doi:10.1109/JSAC.2002.801222.

27. Imai, T., \& Taga, T. (2006). Statistical scattering model in urban propagation environment. IEEE Transactions on Vehicular Technology, 55(4), 1081-1093. doi:10.1109/TVT.2006.877469.

28. Quitin, F., Oestges, C., Horlin, F., \& De Doncker, P. (2009). Multipolarized MIMO channel characteristics: Analytical study and experimental results. IEEE Transactions on Antennas and Propagation, 57(9), 2739-2745. doi:10.1109/TAP.2009.2027041.

29. Ziółkowski, C., \& Kelner, J. M. (2015). Geometry-based statistical model for the temporal, spectral, and spatial characteristics of the land mobile channel. Wireless Personal Communications, 83(1), 631-652. doi:10.1007/s11277-015-2413-3.

30. Andersen, J. B., \& Pedersen, K. I. (2002). Angle-of-arrival statistics for low resolution antennas. IEEE Transactions on Antennas and Propagation, 50(3), 391-395. doi:10.1109/8.999632.

31. Sivasondhivat, K., Takada, J., Ida, I., \& Oishi, Y. (2008). Experimental analysis and site-specific modeling of channel parameters at mobile station in an urban macrocellular environment. IEICE Transactions on Communications, E91-B(4), 1132-1144. doi:10.1093/ietcom/e91-b.4.1132.

32. Abdi, A., Barger, J. A., \& Kaveh, M. (2002). A parametric model for the distribution of the angle of arrival and the associated correlation function and power spectrum at the mobile station. IEEE Transactions on Vehicular Technology, 51(3), 425-434. doi:10.1109/TVT.2002.1002493.

33. Lian, X., Nikookar, H., \& Ligthart, L. P. (2011). Efficient radio transmission with adaptive and distributed beamforming for intelligent WiMAX. Wireless Personal Communications, 59(3), 405-431. doi:10.1007/s11277-011-0237-3.

34. Guillet, J. (2006). Measurement-based statistical model and simulation of mobile station anisotropic power angular spectrum. Electronics Letters, 42(12), 671-673. doi:10.1049/el:20064228.

35. Costa, M., Koivunen, V., \& Poor, H. V. (2014). Estimating directional statistics using wavefield modeling and mixtures of von-Mises distributions. IEEE Signal Processing Letters, 21(12), 1496-1500. doi:10.1109/LSP.2014.2341651. 
36. Ribeiro, C. B., Ollila, E., \& Koivunen, V. (2007). Stochastic maximum-likelihood method for MIMO propagation parameter estimation. IEEE Transactions on Signal Processing, 55(1), 46-55. doi:10.1109/ TSP.2006.882057.

37. Zhang, J., Sheng, N., Zhang, F., Tian, L., Liu, G., Dong, W., et al. (2011). Analysis and modeling of spatial characteristics in urban microscenario of heterogeneous network. EURASIP Journal on Wireless Communications and Networking, 2011(1), 187. doi:10.1186/1687-1499-2011-187.

38. Zhu, S., Ghazaany, T. S., Jones, S. M. R., Abd-Alhameed, R. A., Noras, J. M., Van Buren, T., et al. (2014). Probability distribution of Rician K-factor in urban, suburban and rural areas using real-world captured data. IEEE Transactions on Antennas and Propagation, 62(7), 3835-3839. doi:10.1109/TAP. 2014.2318072.

39. Pei, F., Zhang, J., \& Pan, C. (2013). Elevation angle characteristics of urban wireless propagation environment at $3.5 \mathrm{GHz}$. In Proceedings of 78th vehicular technology conference, VTC-2013-Fall (pp. 1-5). doi:10.1109/VTCFall.2013.6692178.

40. Lu, Y., Zhang, J., Gao, X., Zhang, P., \& Wu, Y. (2007). Outdoor-indoor propagation characteristics of peer-to-peer system at $5.25 \mathrm{GHz}$. In Proceedings of 66th vehicular technology conference, VTC-2007Fall (pp. 869-873). Presented at the Vehicular Technology Conference, 2007. VTC-2007 Fall. 2007 IEEE 66th. doi:10.1109/VETECF.2007.190.

41. Bronshtein, I. N., Semendyayev, K. A., Musiol, G., \& Mühlig, H. (2007). Handbook of mathematics (5th ed.). Berlin: Springer.

42. Matthews, P. A., Molkdar, D., \& Mohebbi, B. (1989). Direction of arrival and frequency response measurements at UHF. In Proceedings of 5th international conference on mobile radio and personal communications (pp. 43-47).

43. Pedersen, K. I., Mogensen, P. E., \& Fleury, B. H. (1998). Spatial channel characteristics in outdoor environments and their impact on BS antenna system performance. In Proceedings of 48th IEEE vehicular technology conference, IEEE VTC-1998 (Vol. 2, pp. 719-723). doi:10.1109/VETEC.1998. 683676.

44. Takada, J., Fu, J., Zhu, H., \& Kobayashi, T. (2002). Spatio-temporal channel characterization in a suburban non line-of-sight microcellular environment. IEEE Journal on Selected Areas in Communications, 20(3), 532-538. doi:10.1109/49.995512.

45. Fleury, B. H., Tschudin, M., Heddergott, R., Dahlhaus, D., \& Pedersen, K. I. (1999). Channel parameter estimation in mobile radio environments using the SAGE algorithm. IEEE Journal on Selected Areas in Communications, 17(3), 434-450. doi:10.1109/49.753729.

46. Mogensen, P. E., Pedersen, K. I., Leth-Espensen, P., Fleury, B. H., Frederiksen, F., Olesen, K., \& Larsen, S. L. (1997). Preliminary measurement results from an adaptive antenna array testbed for GSM/ UMTS. In Proceedings of 47th IEEE vehicular technology conference, IEEE VTC-1997 (Vol. 3, pp. 1592-1596). doi:10.1109/VETEC.1997.605826.

47. Kuchar, A., Taferner, M., Tangemann, M., \& Hoek, C. (1999). Field trial with a GSM/DCS1800 smart antenna base station. In Proceedings of 50th IEEE vehicular technology conference, IEEE VTC-1999Fall (Vol. 1, pp. 42-46). doi:10.1109/VETECF.1999.797037.

48. Failli, M. (1989). COST 207. Digital land mobile radio communications (No. Final report COST 207 (1984.03.14.-1988.09.13)). Luxembourg City, Luxembourg: Commission of the European Communities, Directorate-General Telecommunications, Information Industries and Innovation.

49. Rohatgi, A. (2015). WebPlotDigitizer 3.8. Retrieved from http://arohatgi.info/WebPlotDigitizer/.

50. Piechocki, R. J., Tsoulos, G. V., \& McGeehan, J. P. (1998). Simple general formula for PDF of angle of arrival in large cell operational environments. Electronics Letters, 34(18), 1784-1785. doi:10.1049/el: 19981264.

51. Liberti, J. C., \& Rappaport, T. S. (1996). A geometrically based model for line-of-sight multipath radio channels. In Proceedings of 46th IEEE vehicular technology conference, IEEE VTC-1996. Mobile technology for the human race (Vol. 2, pp. 844-848). doi:10.1109/VETEC.1996.501430.

52. Andrade, A., \& Covarrubias, D. (2003). Radio channel spatial propagation model for mobile $3 \mathrm{G}$ in smart antenna systems. IEICE Transactions on Communications, 86(1), 213-220.

53. Bevan, D. D. N., Ermolayev, V. T., Flaksman, A. G., \& Averin, I. M. (2004). Gaussian channel model for mobile multipath environment. EURASIP Journal on Applied Signal Processing, 2004(9), 1321-1329. doi:10.1155/S1110865704404028.

54. Laurila, J., Molisch, A. F., \& Bonek, E. (1998). Influence of the scatterer distribution on power delay profiles and azimuthal power spectra of mobile radio channels. In Proceedings of 5th IEEE international symposium on spread spectrum techniques and applications (Vol. 1, pp. 267-271). doi:10.1109/ ISSSTA.1998.726240.

55. Shao, J. (2007). Mathematical statistics (2nd ed.). New York: Springer. 

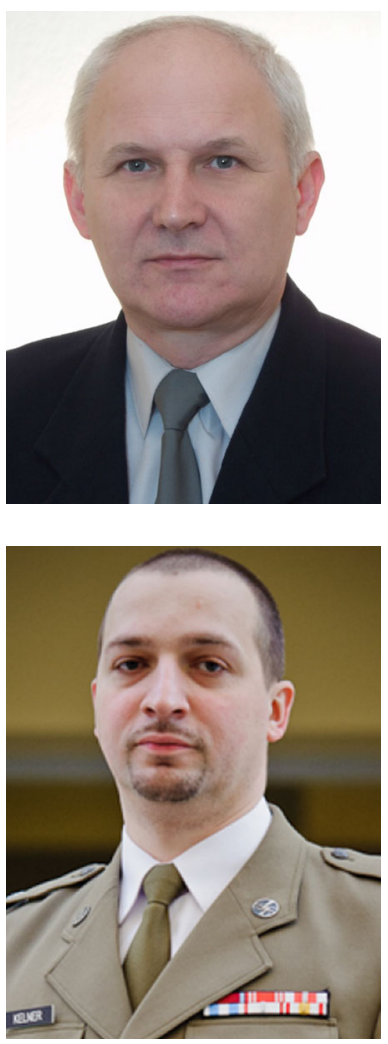

Cezary Ziółkowski was born in Poland in 1954. He received M.Sc. and Ph.D. degrees from the Military University of Technology (MUT), Warsaw, Poland, in 1978 and 1993, respectively, all in telecommunication engineering. In 1989 he received M.Sc. degree from the University of Warsaw in mathematics, specialty-analysis mathematics applications. In 2013 he received the habil. degree (D.Sc.) in Radio Communications Engineering from MUT. From 1982 to 2013 he was a researcher and lecturer while since 2013 he has been a professor of Faculty of Electronics with MUT. He was engaged in many research projects, especially in the fields of radio communications systems engineering, radio waves propagations, radio communication network resources management and electromagnetic compatibility in radio communication systems. He is an author or co-author of over eighty scientific papers and research reports.

Jan M. Kelner was born in Bystrzyca Kłodzka, Poland in 1977. He received his M.Sc. degree in Applied Physics in 2001, his Ph.D. in Telecommunications in 2011, all from the Military University of Technology (MUT) in Warsaw, Poland. In 2011 he won "The Winner Takes All" contest on research grant of MUT Rector, and his Ph.D. thesis won the third prize in the Mazovia Innovator contest. He has authored or co-authored more than seventy articles in peer-reviewed journals and conferences. He is a reviewer for four scientific journals and two conferences. He works as an assistant professor in the Institute of Telecommunications, in the Faculty of Electronics of MUT. His current research interests include wireless communications, simulations, modelling, and measurements of channels and propagation, signal-processing, navigation and localization techniques. 